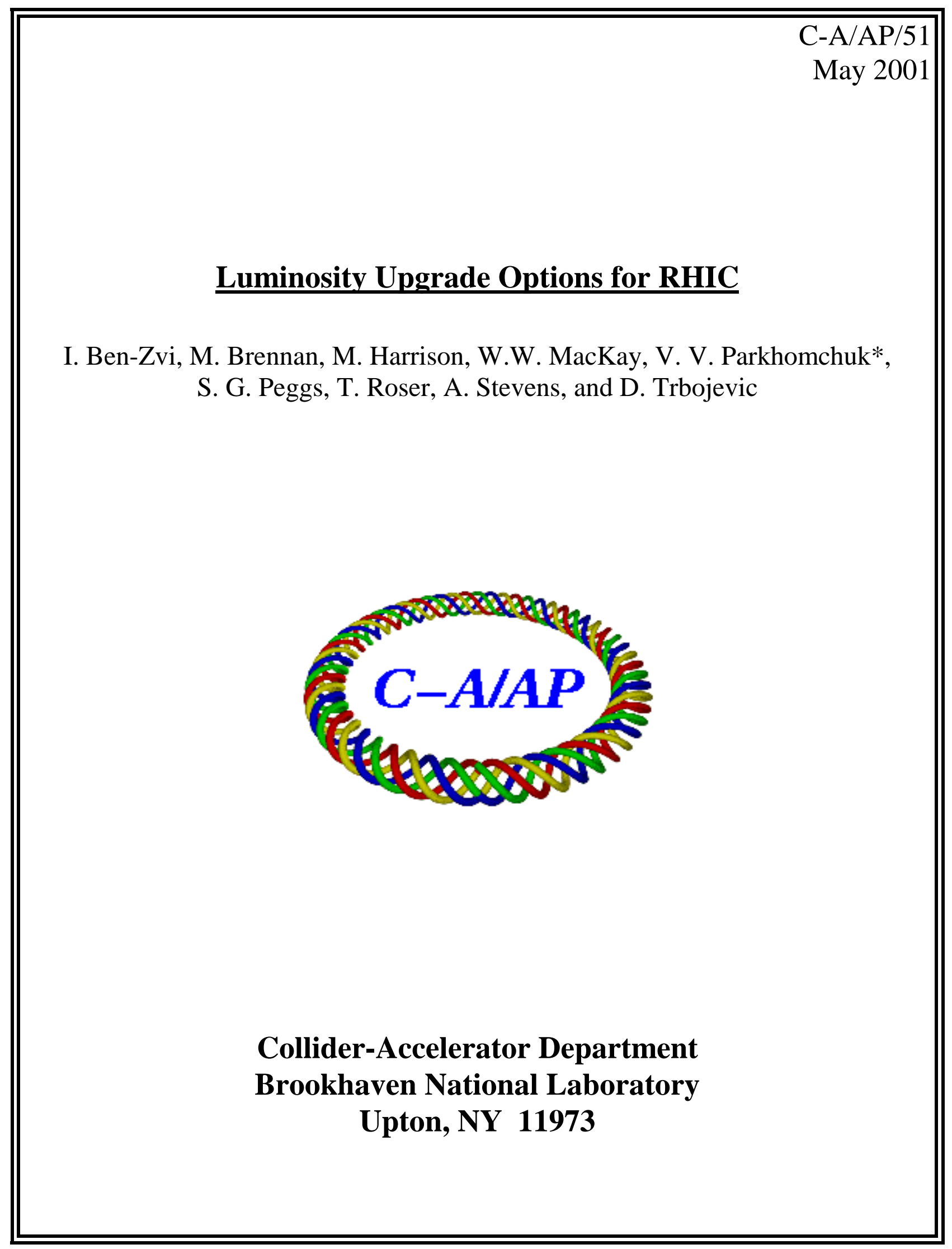




\title{
Luminosity Upgrade Options for RHIC
}

\author{
I. Ben-Zvi, M. Brennan, M. Harrison, W. W. MacKay, V. V. Parkhomchuk*, \\ S. G. Peggs, T. Roser, A. Stevens, and D. Trbojević
}

November 15, 2000

\section{Contents}

1 Executive Summary $r$

2 Beam-Beam Considerations $r$

2.1 Single bunch intensity limit . . . . . . . . . . . . . . . . . 5

2.2 Luminosity performance at the beam-beam and angular aperture limits . . . . . . . . . 6

2.3 Instabilities . . . . . . . . . . . . . . . . . . . . . . . 8

3 Electron Cooling $\quad \mathbf{8}$

3.1 RHIC performance at top energy, without cooling . . . . . . . . . . . . . . 9

3.2 RHIC performance at top energy, with electron cooling . . . . . . . . . . . . . . 9

3.3 The recombination and lifetime of ions . . . . . . . . . . . . . . . . 10

3.4 Collision losses . . . . . . . . . . . . . . . . . . . . 12

4 Conclusions and comments $r$

$\begin{array}{ll}\text { Appendix A: } & 14\end{array}$

A.1 Radio Frequency system . . . . . . . . . . . . . . . . . . . . . 15

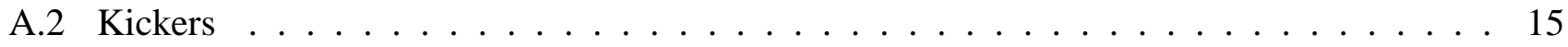

A.3 Long range beam-beam interactions . . . . . . . . . . . . . . . . . . 16

A.4 Cryogenic beam pipe and BPM signal cable heating . . . . . . . . . . . . . . . 16

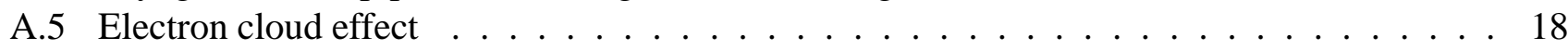

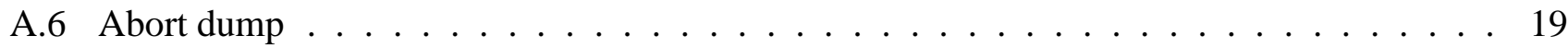

A.7 Instabilities . . . . . . . . . . . . . . . . . . . . . . . . 19

$\begin{array}{lr}\text { References } & \mathbf{2 0}\end{array}$

*Budker Institute of Nuclear Physics, Novosibirsk 630090, Russia 


\section{Executive Summary}

The RHIC lattice allows for simultaneous operation at six different interaction region, each with a design luminosity of $2 \times 10^{26} \mathrm{~cm}^{-2} \mathrm{~s}^{-1}$ for gold beams. It is expected that this design luminosity will be reached during the FY2001 heavy ion run. The machine parameters are shown in Table 1 in column "RDM" (RHIC Design Manual).

\begin{tabular}{|lcccc|}
\hline Scheme & Units & RDM & RDM+ & RHIC II \\
& & & & \\
\hline Initial Emittance $(95 \%), \epsilon$ & {$[\pi \mu \mathrm{m}]$} & 15 & 15 & 15 \\
Final Emittance $(95 \%), \epsilon$ & {$[\pi \mu \mathrm{m}]$} & 40 & 40 & $<6$ \\
IP beta function, $\beta^{*}$ & {$[\mathrm{~m}]$} & 2.0 & 1.0 & 1.0 \\
Number of bunches, $M$ & & 60 & 120 & 120 \\
Bunch population, $N$ & {$\left[10^{9}\right]$} & 1.0 & 1.0 & 1.0 \\
& & & & \\
Beam-beam parameter per IR, $\xi$ & & .0016 & .0016 & .004 \\
Angular beam size, $\sigma^{\prime *}$ & {$\left[\mu \mathrm{rad}^{*}\right]$} & 108 & 153 & 95 \\
RMS beam size, $\sigma^{*}$ & {$\left[\mu \mathrm{m}^{*}\right.$} & 216 & 150 & 95 \\
& & & & \\
Peak Luminosity, $L_{0}$ & {$\left[10^{27} \mathrm{~cm}^{-2} \mathrm{~s}^{-1}\right]$} & 0.8 & 3.2 & 8.3 \\
Average Luminosity, $\langle L\rangle$ & {$\left[10^{27} \mathrm{~cm}^{-2} \mathrm{~s}^{-1}\right]$} & 0.2 & 0.8 & 7 \\
& & & & \\
\hline
\end{tabular}

Table 1: The luminosity performance of RHIC in scenarios of Au+Au collisions at $100 \mathrm{GeV} /$ nucleon. The luminosity averages given for "RDM" and "RDM+" are averaged over a 10 hour store. For the "RHIC II" scenario luminosity is averaged over 5 hours due to the beam-beam burn-off from actual collisions.

A first upgrade of the luminosity by about a factor of four consists of increasing the number of bunches from about 60 to about 120 and decreasing $\beta^{*}$ from $2 \mathrm{~m}$ to $1 \mathrm{~m}$. This will not require any substantial new hardware. However, due to the larger beam size in the interaction triplets the non-linear local correction elements will have to be carefully optimized. It is expected that this level of performance can be reached during the FY2003 running period. The machine parameters for this enhanced luminosity are shown in column "RDM+".

A further increase of the number of bunches (Appendix A) or decrease of $\beta^{*}$ is possible and has been studied. However, it would require substantial upgrades or modifications of the collider detectors. The former will reduce the time interval between collisions to less than $100 \mathrm{~ns}$ and the latter would require additional triplets close to the collision point.

Alternatively the luminosity can be enhanced by increasing the number of ions per bunch or by decreasing the transverse emittance of the beam. However, already at the present bunch intensity and beam emittance the luminosity is expected to decrease very rapidly during a store due to intrabeam scattering (IBS). This is the reason for the large difference between peak and average luminosity in Table 1. To overcome this limitation we are proposing to counteract intrabeam scattering by electron cooling the gold beams at storage energy. 
Cooling the gold beams at $100 \mathrm{GeV} /$ nucleon requires an electron beam energy of about $50 \mathrm{MeV}$ and an average beam current of about $10 \mathrm{~mA}$. The electron accelerator would be a superconducting, energyrecuperating linac very similar to an existing linac[1] operating for a free electron laser at TJNAF. With electron cooling the beam emittance can be reduced and maintained throughout the store and the luminosity increased until non-linear effects of the two colliding beams on each other limit any further increase (beam-beam limit). With the parameters shown in Table 1 in column "RHIC II" a 35-fold luminosity increase over RHIC design luminosity could eventually be achieved. The RHIC electron cooler could be completed by FY2005.

Upgrading the heavy ion beam from gold to uranium ions at similar bunch intensities will be possible using the Electron Beam Ion Source (EBIS) which is presently in development.

\section{Luminosity upgrades for polarized proton operation}

The RHIC spin physics program uses the unique capability of RHIC to accelerate and collide polarized proton beams at a center-of-mass energy of up to $500 \mathrm{GeV}$ and a luminosity of up to $2 \times 10^{32} \mathrm{~cm}^{-2} \mathrm{~s}^{-1}$. Although the physics potential of this capability still needs to be exploited there are upgrades to RHIC that can significantly extend the physics reach of this program.

Since the spin physics program relies on high precision measurements a luminosity upgrade is most useful. The proton beam intensity can be increased, or the beam emittance be decreased until the beambeam limit is reached which corresponds to a luminosity of about $4 \times 10^{32} \mathrm{~cm}^{-2} \mathrm{~s}^{-1}$. The RHIC electron cooler proposed for heavy ion operation could be used at injection energy to achieve this reduction of the proton beam emittance. The machine parameters for the expected luminosity during FY2001 (column "RDM"), the enhanced luminosity as discussed above for gold beams (column "RDM+"), and the luminosity at the beam-beam limit (column "RHIC II") are listed in Table 2.

\begin{tabular}{|lcccc|}
\hline Scheme & Units & RDM & RDM+ & RHIC II \\
\hline Emittance (95\%), $\epsilon$ & & & & \\
IP beta function, $\beta^{*}$ & {$[\pi \mu \mathrm{m}]$} & 20 & 20 & 12 \\
Number of bunches, $M$ & {$[\mathrm{~m}]$} & 2.0 & 1.0 & 1.0 \\
Bunch population, $N$ & {$\left[10^{11}\right]$} & 60 & 120 & 120 \\
& & 1.0 & 2.0 & 2.0 \\
Beam-beam parameter per IR, $\xi$ & {$[\mu \mathrm{rad}]$} & 79 & 112 & 86 \\
Angular beam size, $\sigma^{\prime *}$ & {$\left[\mu \mathrm{m}^{*}\right]$} & 158 & 112 & 86 \\
RMS beam size, $\sigma^{*}$ & & & & \\
& {$\left[10^{31} \mathrm{~cm}^{-2} \mathrm{~s}^{-1}\right]$} & 1.5 & 24 & 40 \\
Peak Luminosity, $L_{0}$ & & & & \\
\hline
\end{tabular}

Table 2: The luminosity performance of RHIC in scenarios of $p+p$ collisions at $250 \mathrm{GeV}$ per beam. Note that for the RHIC II and RDM+ scenarios, we have assumed that beams are colliding at only two or three IR's respectively, so the total tune shift limit is still 0.024 . 
It also seems possible to install in one or two interaction regions an additional pair of high field focusing triplets that would reduce beta-star to about $30 \mathrm{~cm}$ increasing the luminosity by an additional factor of 3 . Finally, the number of bunches in each ring could be increased from 120 to 360 increasing the luminosity by another factor of 3. The latter two upgrade options would also require upgrades to the detectors. Taken together these upgrades would allow for a polarized proton luminosity at $500 \mathrm{GeV}$ of up to $4 \times$ $10^{33} \mathrm{~cm}^{-2} \mathrm{~s}^{-1}$, a 20 -fold increase over the present luminosity goal for $\mathrm{pp}$.

It may also be interesting to increase the center-of-mass energy of the polarized proton collisions. The arc dipoles and quadrupoles in RHIC have a margin of about $30 \%$ beyond the operating field for 250 $\mathrm{GeV}$ beam energy. Exploiting this margin would allow for operation at a center-of-mass energy of about $650 \mathrm{GeV}$. One or two interaction regions would have to be refitted with higher field magnets to produce collisions whereas the remaining interaction regions could be retuned for simply transporting the higher energy beam without producing collisions. 


\section{Beam-Beam Considerations}

The RMS ion beam size at the interaction point (IP) is written as

$$
\sigma^{*}=\sqrt{\frac{\beta^{*} \epsilon}{6 \pi(\beta \gamma)}}
$$

where $\epsilon$ is the normalized $95 \%$ emittance, and $(\beta \gamma)$ is a Lorentz factor. A closely related quantity, the RMS angular beam size, is simply given by

$$
\sigma^{*}=\frac{\sigma^{*}}{\beta^{*}}
$$

if it is (reasonably) assumed that $\alpha^{*}=0$ at the IP. It is also assumed, for the sake of simplicity, that both ion beams are round at the IP - the horizontal and vertical emittances, beta functions, and beam sizes are assumed to be identical. Finally, it is assumed that all bunches have the same intensity, whichever ring they occupy. With these assumptions, the beam-beam parameter $\xi$ is given by

$$
\xi=N \frac{r \beta^{*}}{4 \pi \gamma \sigma^{* 2}}=\frac{3 r}{2} \frac{N}{\epsilon}
$$

where $N$ is the number of ions per bunch, and the relativistic limit $(\beta \approx 1)$ has been assumed. The classical radius of the ion

$$
r=\frac{Z^{2}}{A} \frac{e^{2}}{4 \pi \epsilon_{0}} \frac{1}{M_{0} c^{2}}
$$

depends on the atomic number $Z$ (79 for gold), the atomic mass number $A$ (197 for gold), and the mass per nucleon, $M_{0}$. Its value is $r_{A u}=49.0 \times 10^{-18} \mathrm{~m}$ for gold, and $r_{p}=1.53 \times 10^{-18} \mathrm{~m}$ for protons.

\subsection{Single bunch intensity limit}

Eq. 3 places a fundamental limit on the single bunch phase space density $N / \epsilon$, since $\xi$ has a critical maximum value which cannot be surpassed, due to nonlinear dynamics. Note that neither the beta function $\beta^{*}$ nor the energy $\gamma$ enter Eq. 3 - the single bunch intensity limit cannot be enhanced or reduced by optics or energy upgrades. Also note that $\xi$ is a "beam-beam" parameter, not a "tune shift" parameter - if there are $N_{\text {ip }}$ head-on collisions per turn, a small amplitude particle suffers a total tune shift of

$$
\Delta Q=N_{\text {ip }} \xi
$$

The exact critical maximum value $\xi_{c}$ depend on many details such as the number of head-on collisions per turn, the presence of long range beam-beam interactions, the betatron and synchrotron tunes, the chromaticity, the possible presence of external sources of tune modulation, damping, etc. Even without a detailed model of an upgraded RHIC, it is reasonable to assume that

$$
\xi_{c} \approx \frac{0.024}{N_{\mathrm{ip}}}
$$

an approximate value which is justified not only by general calculations and simulations, but also by direct experience at the SPS[2], and the Tevatron[3, 4]. It is unlikely that electron cooling with characteristic times longer than about 1 second will significantly enhance this value $[5,6]$. 
The single bunch intensity limit due to the beam-beam interaction is directly proportional to the emittance, which is nominally expected to increase from about $15 \pi \mu \mathrm{m}$ to $40 \pi \mu \mathrm{m}$ in the course of a 10 hour store, due to intrabeam scattering (IBS) [7]. Inverting Eq. 3, and using nominal numerical values for $\xi_{c}$ and $\epsilon$, the maximum single bunch gold intensity is found to be

$$
N_{c}=2.6 \times 10^{9}\left(\frac{\epsilon}{15 \pi[\mu \mathrm{m}]}\right)\left(\frac{\xi_{c}}{0.004}\right) .
$$

This is to be compared with the nominal single bunch intensity of $10^{9}$ ions per bunch quoted in the RHIC Design Manual [7]. The beam-beam limit is not far away.

\begin{tabular}{|lcr|}
\hline Parameter & units & value \\
\hline & & \\
Gold top energy, $E_{A u}$ & {$[\mathrm{GeV} / \mathrm{u}]$} & 100 \\
Gold top energy, $\gamma$ & & 107 \\
Circumference, $C$ & {$[\mathrm{~m}]$} & 3834 \\
Revolution frequency, $f_{\text {rev }}$ & {$[\mathrm{kHz}]$} & 78.3 \\
& & \\
Effective triplet distance, $\hat{d}$ & {$[\mathrm{~m}]$} & 36.0 \\
Triplet bore radius, $a$ & {$[\mathrm{~mm}]$} & 65 \\
Minimum triplet aperture, $n$ & {$[\sigma]$} & 8 \\
& & \\
Maximum angular beam size, $\sigma_{c}^{* *}$ & {$[\mu \mathrm{rad}]$} & 226 \\
Critical beam-beam parameter, $\xi_{c}$ & & 0.004 \\
& & \\
\hline
\end{tabular}

Table 3: Primary parameters for the as-built RHIC configuration for heavy ion collisions, including interaction region optics.

\subsection{Luminosity performance at the beam-beam and angular aperture limits}

The luminosity per interaction point is given by the equation

$$
L=f_{\mathrm{rev}} \frac{M N^{2}}{4 \pi \sigma^{* 2}}
$$

where $f_{\text {rev }}$ is the revolution frequency, and $M$ is the number of bunches in each beam. One way to reparameterize this equation gives

$$
L=M \xi^{2} \sigma^{* 2}\left(\frac{4 \pi f_{\mathrm{rev}} \gamma^{2}}{r^{2}}\right)
$$

where the term in parentheses is constant at fixed energy. This parameterization is appropriate when the maximum luminosity is simultaneously limited - or nearly limited - by beam-beam effects and by interaction region optics, since then the values of $\xi$ and $\sigma^{\prime *}$ are well known. 
The beta function at a distance $d$ from the IP, still in the drift region before the first quadrupole, is given by

$$
\beta(d)=\beta^{*}+\frac{d^{2}}{\beta^{*}} \approx \frac{d^{2}}{\beta^{*}} .
$$

where the approximation, good if $d \gg \beta^{*}$, is usually valid. Similarly, the maximum value of $\hat{\beta}$ in the interaction region triplet is inversely proportional to the value of $\beta^{*}$. By analogy with Eq. 10, this relationship is conveniently described by introducing the nearly constant "effective triplet distance" $\hat{d}$, which is defined by

$$
\hat{d}=\sqrt{\hat{\beta} \beta^{*}} .
$$

The effective triplet distance also relates the angular beam size at the IP to the maximum beam size $\hat{\sigma}$, since

$$
\hat{\sigma}=\hat{d} \sigma^{*} .
$$

The upper limit of this maximum beam size is constrained by the requirement of an aperture at least $n \approx 8$ times the rms size of the beam in the triplet quadrupoles. Thus, the angular beam size at the IP must be less than a critical value $\sigma_{c}^{\prime *}$ which is proportional to the "effective angular aperture" of the triplet, $a / \hat{d}$, through

$$
\sigma^{*} \leq \sigma_{c}^{*}=\frac{1}{n} \frac{a}{\hat{d}} .
$$

Note that the critical value $\sigma_{c}^{\prime *}$ is independent of emittance for non-pathological values of $\beta^{*}$. The effective angular aperture is the principal figure of merit measuring the potency of IR optics schemes. It is improved by using larger bore quadrupoles (increasing $a$ ) or by moving the triplet closer to the IP (decreasing $\hat{d}$ ).

Table 3 lists some basic design parameters for RHIC including the effective triplet distance and the bore radius of the triplet quadrupoles. This leads to a maximum angular beam size of $\sigma_{c}^{\prime *}=0.226 \mathrm{mrad}$, a limit which will be slightly violated if a gold beam with an emittance of $\epsilon=40 \pi \mu \mathrm{m}$ is stored in a lattice with $\beta^{*}=1.0 \mathrm{~m}$. Putting Table 3 values into Eq. 9 yields

$$
L=\frac{M}{120}\left(\frac{\xi}{.004}\right)^{2}\left(\frac{\sigma^{\prime *}}{.226[\mathrm{mrad}]}\right)^{2} 4.6 \times 10^{28}\left[\mathrm{~cm}^{-2} \mathrm{~s}^{-1}\right] .
$$

Table 4 shows the performance of RHIC for the following 3 different sets of parameters, all with the current IR magnet configuration:

1. The first column, labeled "RDM", shows the values recorded in the Reference Design Manual.

2. Column "RDM+" represents an early upgrade to $M=120$ bunches with $\beta^{*}=1.0 \mathrm{~m}$. No major difficulties are foreseen.

3. Column "RHIC II" adds electron cooling to reduce the emittance and counteract blowup from IBS.

Appendix A discusses some of the issues for surpassing "RHIC II" with higher currents and even more bunches. 


\begin{tabular}{|lccc|}
\hline Scheme & RDM & RDM+ & RHIC II \\
& & & \\
\hline & & & \\
Emittance $(95 \%), \epsilon[\pi \mu \mathrm{m}]$ & 15 & 15 & 5.8 \\
IP beta function, $\beta^{*}[\mathrm{~m}]$ & 2.0 & 1.0 & 1.0 \\
Number of bunches, $M$ & 60 & 120 & 120 \\
Bunch population, $N\left[10^{9}\right]$ & 1.0 & 1.0 & 1.0 \\
& & & \\
Beam-beam parameter, $\xi$ & .0016 & .0016 & .004 \\
Angular beam size, $\sigma^{\prime *}[\mu \mathrm{rad}]$ & 108 & 153 & 95 \\
RMS beam size, $\sigma^{*}[\mu \mathrm{m}]$ & 216 & 153 & 95 \\
& & & \\
Peak Luminosity, $L\left[10^{27} \mathrm{~cm}^{-2} \mathrm{~s}^{-1}\right]$ & 0.8 & 3.2 & 8.3 \\
Average Luminosity $\langle L\rangle\left[10^{27} \mathrm{~cm}^{-2} \mathrm{~s}^{-1}\right]$ & 0.2 & 0.8 & 8.3 \\
& & & \\
\hline
\end{tabular}

Table 4: The luminosity performance of RHIC for $\mathrm{Au}-\mathrm{Au}$ collisions in 3 scenarios, at or near the beambeam and angular aperture limits, with the current IR optics. Electron cooling is assumed for the "RHIC II" scenario.

\subsection{Instabilities}

The scenarios "RDM" and "RDM+" have been studied[20] in some detail for impedances and instabilities and are not expected to cause much trouble; although, dampers may be required at injection. The "RHIC II" scenario has the same beam current and number of bunches as "RDM+" with only a smaller emittance and larger beam-beam parameter. We expect that this factor of 2.5 increase in the beam-beam parameter will still be manageable with the designed damper systems. As we gain more experience with the running machine in the next couple of years, we should be able to gain confidence in our model of impedances in RHIC.

\section{Electron Cooling}

Since electron cooling[21] was proposed by G.I. Budker in 1966 it has been successfully implemented in many places, beginning in Novosibirsk. All coolers built to date use a relatively low energy electron beam produced by an electrostatic accelerator.

While the cooling process in the co-moving reference frame of the ions is not affected by the ion energy, higher ion energies require that the electron energy of the cooler be much higher. For example, RHIC's $100 \mathrm{GeV} /$ nucleon requires about $50 \mathrm{MeV}$ electrons. The accelerator techniques which have to be used for the electrons are different. Other differences are that all coolers so far were operated in storage rings, not colliders. Colliders provide new opportunities and new challenges for electron cooling.

We foresee that the electron accelerator for the RHIC cooler will be a superconducting, energy recuperating electron linac. Such an accelerator has been operated for an FEL at Jefferson Laboratory with great success[1]. The energy is about $50 \mathrm{MeV}$ and an average current of $5 \mathrm{~mA}$ has been achieved with a phenomenal energy recovery in the linac, better than $99.99 \%$ (the low energy injector power is not recovered). 
Table 5: Basic parameters assumed for electron cooling scenario.

\begin{tabular}{|lccc|}
\hline & Injection & Beginning of store & End of store* \\
\hline Nominal bunch intensity & $1 \times 10^{9}$ & $1 \times 10^{9}$ & $<1 \times 10^{9}$ \\
Transverse emittance, $\epsilon_{95 \%}^{N}[\pi \mu \mathrm{m}]$ & 10 & 15 & 40 \\
rms bunch length, $\sigma_{z}[\mathrm{~m}]$ & .47 & 0.12 & 0.2 \\
rms momentum spread, $\sigma_{p} / p$ & 0.00027 & 0.00053 & 0.0009 \\
\hline
\end{tabular}

${ }^{*}$ End-of-Store parameters are for the simulation without electron cooling.

Since the cooling rate increases rapidly for ions as they reach equilibrium, the core of the beam might become too dense. By slightly modulating the electron (either in momentum or phase) the core of the distribution may be smeared to achieve a better shape. It might be possible cool past the beam-beam limit of 0.004 by modulating the cooling so that the charge distribution is flattened in phase space thus reducing the space-charge tune spread.

\subsection{RHIC performance at top energy, without cooling}

The results of the following preliminary calculations are taken from a simple one-particle simulation program. Various beam parameters were calculated as a function of storage time at top energy with no electron cooling. The simulation of beam heating (increase of transverse and longitudinal ion beam emittances) is based on a simple IBS model[22] and a random oscillation of the colliding ion beam at the interaction point with an amplitude of 0.01 microns. Ions are considered to be lost when they escape the longitudinal bucket. Here the fraction of ions left in the bunch is approximated by the ratio of the bucket area to the emittance,

$$
\eta_{\mathrm{N}}=\frac{2 \epsilon_{l 0}}{\epsilon_{l}+\epsilon_{l 0}}, \quad \text { if } \epsilon_{l}>\epsilon_{l 0} .
$$

If cooling is applied so the longitudinal emittance is decreasing, then $\epsilon_{l}<\epsilon_{l 0}$, and this ratio would be one.

In Fig. 1 we see that, during 10 hours of storage starting with "RDM+" parameters, there is an increase in emittance of a factor of 2 to 3 , in agreement with the nominal values given in the RHIC parameter table. This is the effect of intrabeam scattering (IBS). The change in emittances and particle loss lead to a luminosity which decreases as a function of storage time as shown in Fig. 2.

\subsection{RHIC performance at top energy, with electron cooling}

In this section we observe the effect of electron cooling on stored beam in RHIC at top energy. This is the simplest and possibly the main scenario of electron cooling at RHIC, in which the cooling is switched on at the beginning of beam storage at top energy. We assume an electron beam with an average current of $9.6 \mathrm{~mA}$, peak current of $0.6 \mathrm{~A}$, and rms bunch length of $20 \mathrm{~cm}$.

During the cooling process, the rms transverse emittance decreases (See Fig. 3.) from $1 \mu \mathrm{m}$ to $0.5 \mu \mathrm{m}$, and the luminosity increases (See Fig. 4.) from the initial value of $4 \times 10^{27} \mathrm{~cm}^{-2} \mathrm{~s}^{-2}$ to $9 \times 10^{27} \mathrm{~cm}^{-2} \mathrm{~s}^{-1}$. Later on we observe a decrease in luminosity due to ion loss as a result of recombination. The beam-beam parameter increases from the initial value 0.002 to 0.004 . The integrated luminosity over the storage time of 10 hours is $300 \mu \mathrm{b}$, or a gain of 6 over the integrated luminosity without cooling.

In this report, the MATHCAD code cooling simulation is just a single particle version; the advantage is very fast response, the disadvantage is large fluctuations near equilibrium. This version is useful for fast 

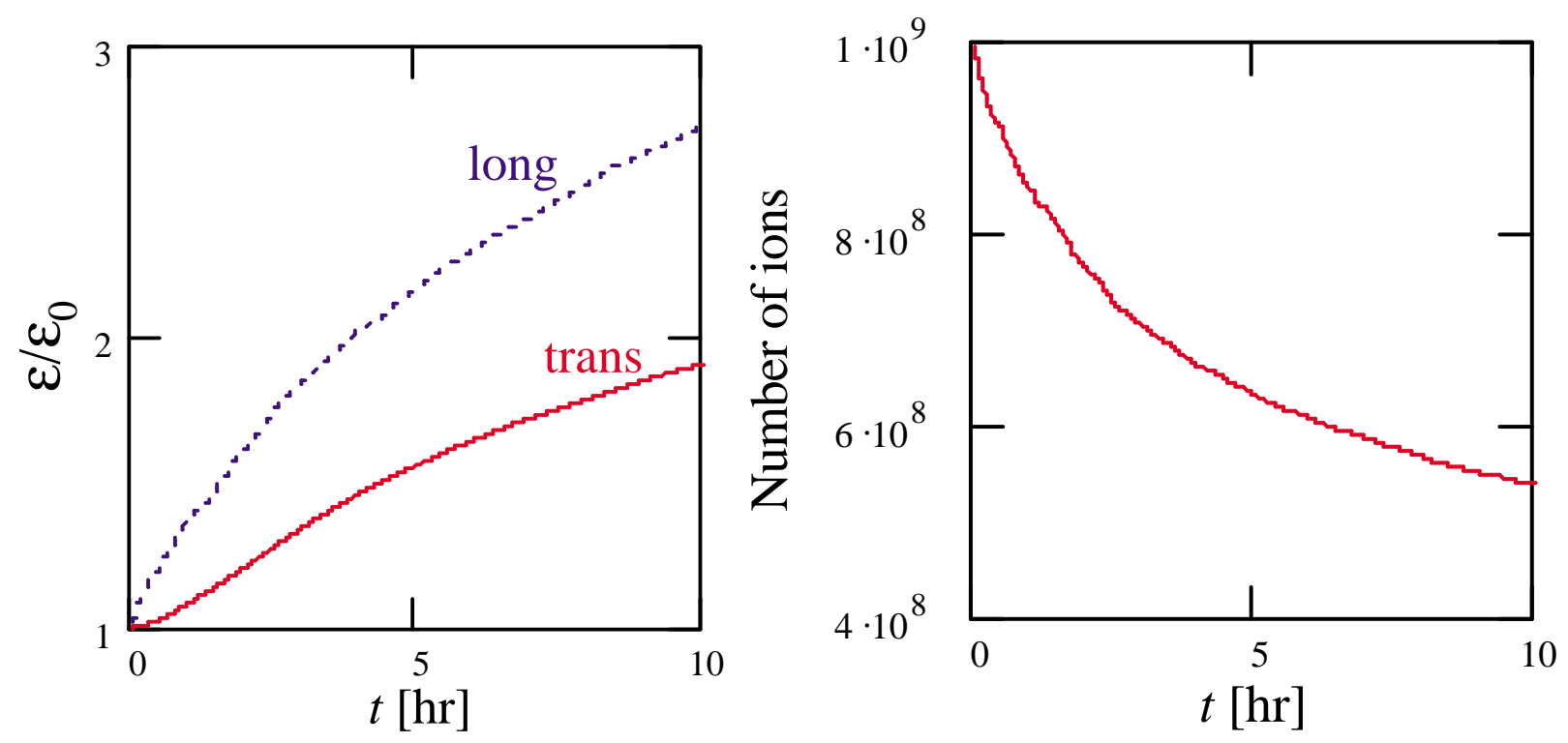

Figure 1: The left figure shows the evolution of emittance blowup from IBS for stored beams with no cooling. In the right plot, the loss of ions from the bunch is the result of particles escaping the longitudinal bucket as explained in the text. For an uncooled RHIC, the integrated luminosity over a single 10 hour run is $50 \mu \mathrm{b}$ (to be compared with $7 \mu \mathrm{b}$ for "RDM"). Initial "RDM+" parameters were assumed.

testing of various parameters. The observation that a very low cooling current $\left(N_{e}=6 \times 10^{9}\right)$ has a useful effect on the RHIC luminosity is a pleasant surprise. A more detailed simulation is currently underway.

\subsection{The recombination and lifetime of ions}

When cooling heavy ions, electrons may recombine with the ions in the region where both electrons and ions are moving together at low relative velocities. This would lead to ion loss from the ring. The theory of radiative recombination was worked out by M. Bell and J. S. Bell[24]. Experiments done at GSI[25] were used for evaluating the recombination coefficient. The Bell recombination equation shows a good agreement with measurements. This is particularly true for fully stripped ions, as is the case for RHIC beams. The effective electron temperature $T$ consists of the direct temperature of the electron beam and an additional component due to the motion of the ions. The effective temperature is

$$
k_{\mathrm{B}} T=k_{\mathrm{B}} T_{e}+\frac{1}{2} m_{e} v_{\perp}^{2}=10^{3} \mathrm{eV}
$$

where the average transverse velocity of the ions is

$$
v_{\perp}=\gamma \beta c \sqrt{\frac{\epsilon}{3 \pi\left\langle\beta_{\text {cool }}\right\rangle}}
$$

and $\left\langle\beta_{\text {cool }}\right\rangle$ is the average of the $\beta$-function through the cooler. The Bell recombination coefficient is given by

$$
\alpha_{\text {rec }}=3.02 \times 10^{-13}\left[\mathrm{~cm}^{3} / \mathrm{s}\right] \frac{Z^{2}}{\sqrt{T}}\left[\ln \left(\frac{11.32 Z^{2}}{\sqrt{T}}\right)+0.14\left(\frac{T}{Z^{2}}\right)^{\frac{1}{3}}\right]
$$



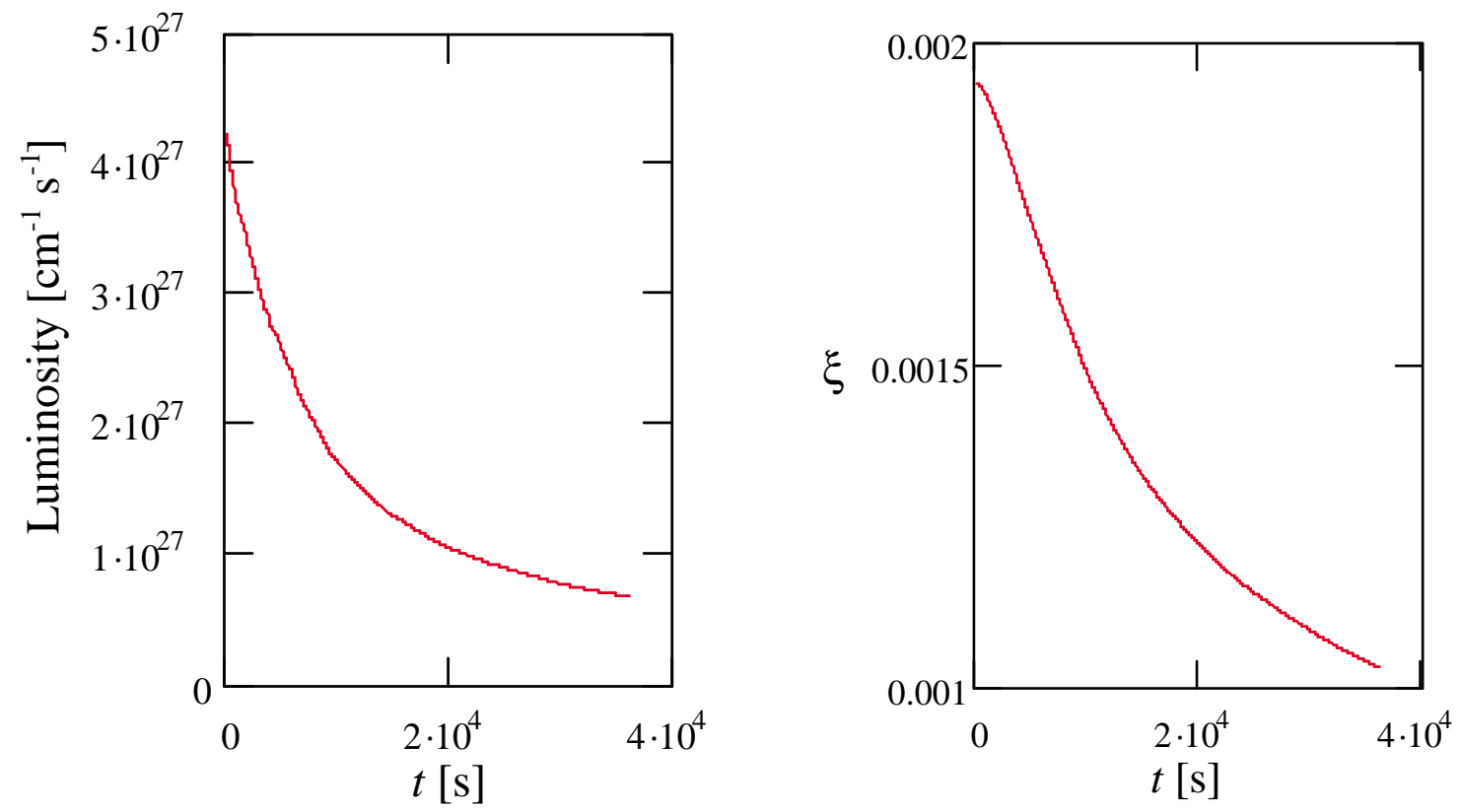

Figure 2: Evolution of luminosity (left) and beam-beam parameter (right) for stored beams with no cooling. Initial "RDM+" parameters were assumed.

with $T$ in eV. For the "RHIC II" scenario, this gives the lifetime due to recombination as

$$
\tau_{\text {rec }}=\frac{\gamma}{n_{e} \alpha_{\text {rec }} \eta_{\text {cool }}}=55 \mathrm{hr}
$$
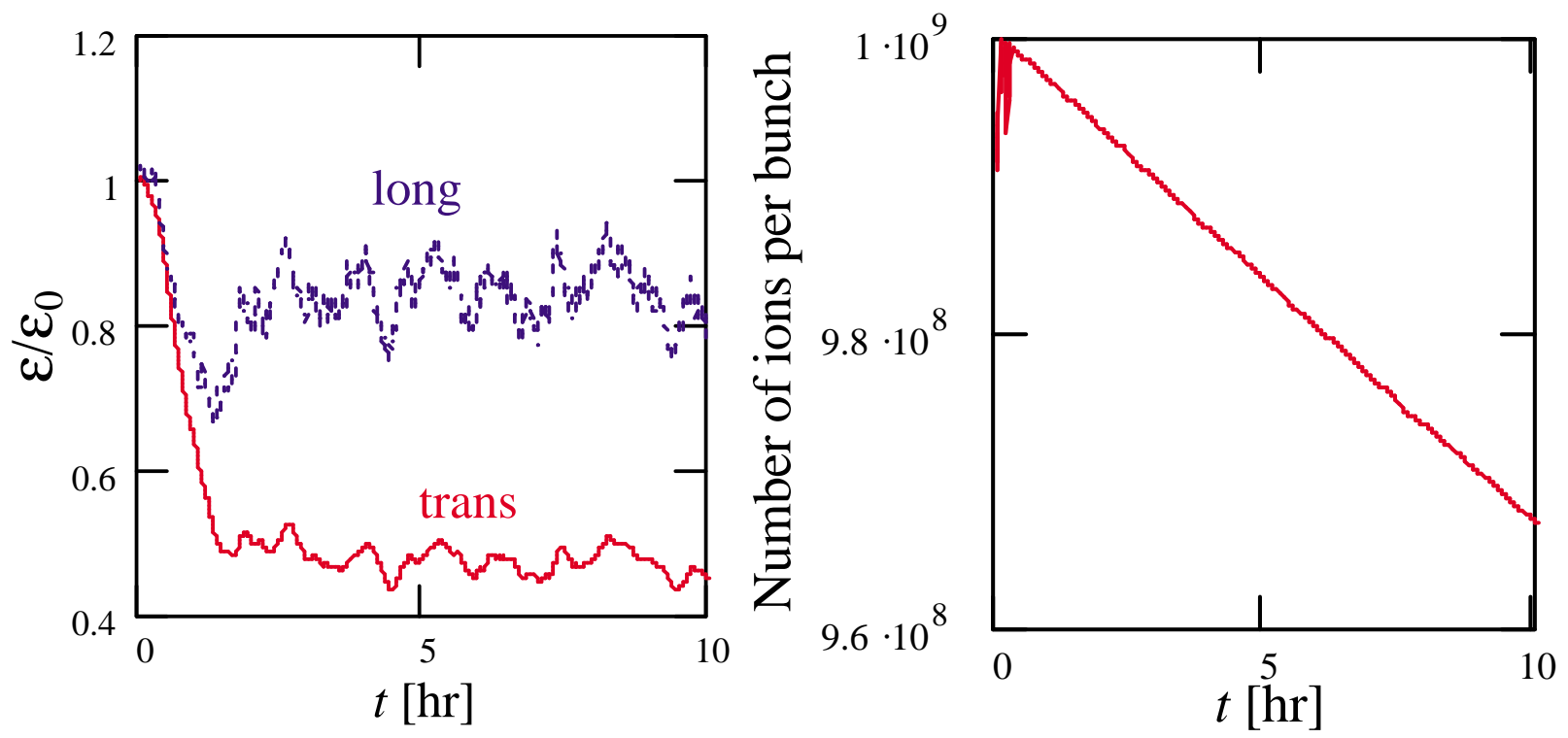

Figure 3: Evolution of emittances (left) and bunch current (right) for stored beams with cooling. This ignores the particle loss from $\mathrm{Au}-\mathrm{Au}$ interactions which is discussed in $\S 3.4$, and would cause a much larger drop in bunch intensity over 10 hours. 

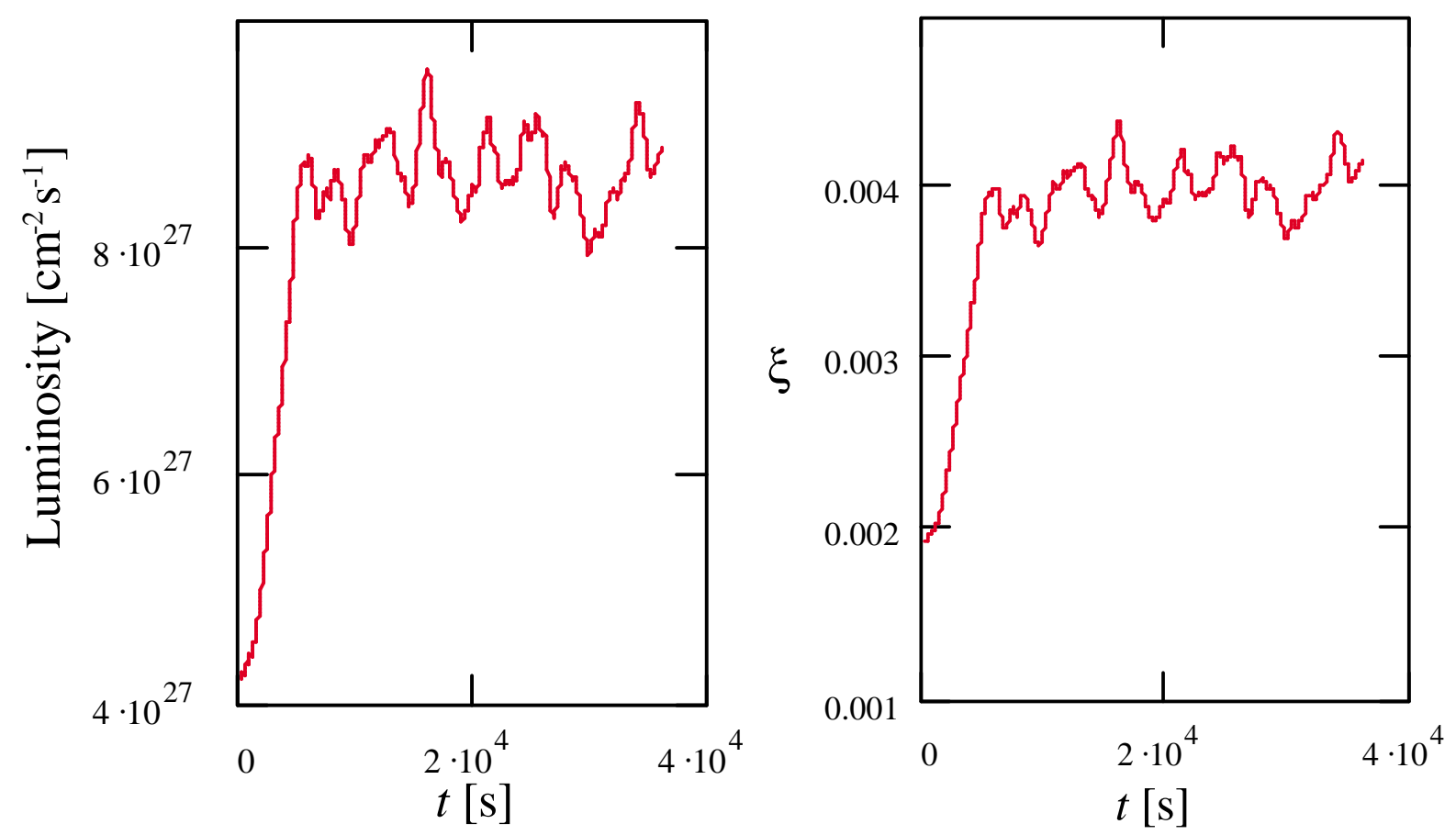

Figure 4: Evolution of luminosity (left) and beam-beam parameter (right) for stored beams with cooling. This ignores the particle loss from Au-Au interactions which is discussed in $\S 3.4$. This loss would actually cause a marked decrease in luminosity and beam-beam parameter over several hours if the emittance and $\beta^{*}$ are held constant.

where $n_{e}$ is the electron beam density, $\alpha_{\text {rec }}$ is taken to be in the rest system of the ion beam, and $\eta_{\text {cool }}$ is the length of the cooling section over the ring circumference.

\subsection{Collision losses}

If we assume constant emittances then the luminosity is proportional to $N^{2}$. With a sufficiently good vacuum the lifetime of a single beam should be several tens of hours. If we ignore losses due to vacuum, instabilities and other single beam effects, then the beam currents will drop only as the particles are used up in collisions.

This loss is dominated by bound electron-positron production and Coulomb dissociation in $\mathrm{Au}-\mathrm{Au}$ collisions. At energies of $100 \mathrm{GeV} /$ nucleon the cross section has been estimated[26] to be

$$
\sigma=\sigma_{\text {pair }}+\sigma_{\text {dis }}=212 \pm 10 \mathrm{~b} .
$$

The rate of collision losses per bunch is given by

$$
\frac{d N}{d t}=-\frac{n_{\mathrm{i}} L(t) \sigma}{M}=-n_{\mathrm{i}} \sigma k N^{2},
$$

where $n_{\mathrm{i}}$ is the number of interaction regions where the bunch collides and

$$
k=\frac{f_{\mathrm{rev}}}{4 \pi \sigma_{x}^{*} \sigma_{y}^{*}} .
$$


Note that in RHIC for most bunches typically $n_{\mathrm{i}}=6$. Solving Eq. 21 and substituting back into the luminosity gives the dependence

$$
f=\frac{L(t)}{L_{0}}=\frac{1}{\left(1+\frac{n_{\mathrm{i}} \sigma L_{0}}{M N_{0}} t\right)^{2}} .
$$

For high luminosity, this decrease in currents due to collisions will decrease the average luminosity as the fill lengthens. With a little more manipulation we see that the average luminosity will fall as

$$
\langle L\rangle=\frac{L_{0}}{1+\frac{n_{i} L_{0} \sigma}{M N_{0}} t} .
$$

In the "RHIC II" scenario with $n_{\mathrm{i}}=6, L_{0}=8.3 \times 10^{27} \mathrm{~cm}^{-2} \mathrm{~s}^{-1}, N=1 \times 10^{9}$ and $M=120$, this gives $\langle L\rangle=3.7 \times 10^{27}$ or $2 \times 10^{27} \mathrm{~cm}^{-2} \mathrm{~s}^{-1}$ respectively, for 4 or 10 hour long stores.

The emittance can be reduced by cooling (reducing the emittance) as the charge per bunch decreases so that the beam-beam parameter remains constant. In this case the average luminosity will still decrease with time but more slowly than with a constant emittance. Recalling Eqs. 3 and 14, we see that the luminosity may be held constant with a constant beam-beam parameter so long as we squeeze the $\beta$-function along with the emittance according to

$$
\beta^{*} \propto \epsilon \propto N \text {. }
$$

If we assume a constant luminosity of $L=8.3 \times 10^{27} \mathrm{~cm}^{-2} \mathrm{~s}^{-1}$ by squeezing $\beta^{*}$ from $1 \mathrm{~m}$ to $0.5 \mathrm{~m}$ and cooling the transverse emittance from about $6 \pi$ to $3 \pi \mu \mathrm{m}$ as the beam is used up, then only half of the particles are left after about 4.7 hours. Allowing for the initial cooling time of about $1.4 \mathrm{hr}$ in Fig. 4, this gives an average luminosity of around $7 \times 10^{27} \mathrm{~cm}^{-2} \mathrm{~s}^{-1}$ over a 4 to 5 hour fill.

\section{Conclusions and comments}

An upgrade in luminosity for $\mathrm{Au}-\mathrm{Au}$ collisions by a factor of 35 beyond the design manual to $7 \times$ $10^{27} \mathrm{~cm}^{-2} \mathrm{~s}^{-1}$ appears to be feasible using 120 bunches of $10^{9}$ ions with electron cooling to reduce the emittance and counteract IBS. The beam emittances would be cooled to increase the beam-beam parameter up to the generally accepted limit of 0.004 for hadron colliders.

The large cross section (212 b) from dissociation and bound electron-positron pair production in $\mathrm{Au}-$ Au collisions give a beam half-life of 4 to 5 hours if the luminosity is held constant by squeezing the $\beta^{*}$ and reducing the transverse emittance in order to retain the beam-beam parameter at 0.004 .

To increase the luminosity beyond a factor of 35 may be possible (See Appendix A.) with major upgrades of the abort system, shielding and damping systems; although this clearly needs more study. Clearly the electron cloud problem may arise as the number of bunches is increased beyond 120 . In addition to impedance issues, the total current may be limited by the heat load on the cryogenics system from induced wall currents.

Electron cooling can be used at injection for polarized protons to reduce the emittance of the beam. We do not propose cooling protons at storage since in this case the IBS is much smaller and would require a higher energy electron beam while being less effective. By steering the beams apart at all but two of the interaction points the beam-beam parameter could be increased by a factor of 3 with the same total tune shift as before. We estimate an initial luminosity of $4 \times 10^{32} \mathrm{~cm}^{-2} \mathrm{~s}^{-1}$ for polarized protons in this case. 


\section{Appendix A: Numbers of Bunches}

In this appendix we include some discussion of further upgrade possiblities with higher bunch intensities and more bunches per ring. Clearly more studies will be necessary to persue any of these scenarios. Calculations of electron cooling have yet to be made; although it is expected cooling will be needed since the effects discussed for the "RHIC II" scenario will be even more severe. Table 6 shows possible upper limits of luminosities for three progressively ambitious scenarios:

1. Column "A" is at the beam-beam limit and at the angular aperture limit of the present interaction region optics, which can achieve a value of $\beta^{*} \approx 0.5 \mathrm{~m}$. Electron cooling will be necessary to maintain the emittance with approximately $2.5 \times 10^{9}$ ions per bunch in the presence of IBS.

2. Column "B" increases the number of bunches to 360 with every $28 \mathrm{MHz}$ bucket filled.

3. Column "C" increases the number of bunches to 2520 with every $197 \mathrm{MHz}$ bucket filled.

\begin{tabular}{|lccc|}
\hline Scheme & A & B & C \\
\hline Emittance $(95 \%), \epsilon[\pi \mu \mathrm{m}]$ & 15 & 15 & 15 \\
IP beta function, $\beta^{*}[\mathrm{~m}]$ & .455 & .455 & .455 \\
Number of bunches, $M$ & 120 & 360 & 2520 \\
Bunch population, $N\left[10^{9}\right]$ & 2.56 & 2.56 & 2.56 \\
& & & \\
Beam-beam parameter, $\xi$ & .004 & .004 & .004 \\
Angular beam size, $\sigma^{\prime *}[\mu \mathrm{rad}]$ & 226 & 226 & 226 \\
RMS beam size, $\sigma^{*}[\mu \mathrm{m}]$ & 103 & 103 & 103 \\
& & & \\
Peak Luminosity, $L\left[10^{27} \mathrm{~cm}^{-2} \mathrm{~s}^{-1}\right]$ & 46 & 140 & 970 \\
& & & \\
\hline
\end{tabular}

Table 6: The luminosity estimates of RHIC for $\mathrm{Au}-\mathrm{Au}$ collisions in 3 scenarios with increased currents and numbers of bunches, at or near the beam-beam and angular aperture limits, with the current IR optics. Calculations have not been made for scenarios $\mathrm{A}, \mathrm{B}$, and $\mathrm{C}$ with electron cooling.

If the total beam current is not limited, the luminosity is directly proportional to $M$, the number of bunches in each beam. What is the maximum number bunches that can be stored?

The RHIC Design Manual [7] quotes a nominal number of 60 ion bunches, but a trivial upgrade to 120 bunches is envisaged. A further increase to 360 bunches is possible in a medium term luminosity upgrade, in which every rf bucket in the $28 \mathrm{MHz}$ acceleration system is filled. Although such an upgrade has a non-trivial impact on some of the existing accelerator and experimental hardware, the technical demands of operating with 360 bunches are reasonable.

Even with 360 bunches, the RHIC bunch spacing of $35.5 \mathrm{~ns}$ is modest by comparison with existing B-factories, and by comparison with the parameters of the LHC and the Tevatron. The SLAC HER has as many as 1658 bunches, with a bunch spacing as small as $4.2 \mathrm{~ns}$. The nominal bunch spacing at the LHC is 
$25 \mathrm{~ns}$, and the Tevatron operates in fixed target mode with bunches spaced by about $18.9 \mathrm{~ns}$. It is therefore natural to consider a much larger number of bunches for RHIC operations.

After acceleration to storage energy, RHIC ion bunches are "rebucketed" by turning off the $28 \mathrm{MHz}$ acceleration system and turning on the $197 \mathrm{MHz}$ storage system, with a harmonic number of $h=7 \times 360=$ 2520. It is natural to consider what happens when every bucket of the storage system is filled. Increasing the number of bunches to 2520 is technically demanding.

Both ends of the spectrum - with a "reasonable"number of $M=360$ bunches, and with a "demanding" number of $M=2520$ bunches - are discussed below. The practical limit is presumably between these two extremes.

The list of bunch or current limitations discussed is not complete. For example, there are significant technical and financial issues associated with increasing the data acquisition rates of the experiments, as the number of bunches (and/or the luminosity) is increased. Also, there is no discussion of the additional amount of radiation protection shielding that might be required.

\section{A.1 Radio Frequency system}

In normal RHIC operations ion bunches are accelerated in the $h=360(28 \mathrm{MHz}) \mathrm{rf}$ acceleration system, and then directly rebucketed into the $h=2520(197 \mathrm{MHz})$ storage system. The nominal rebucketing procedure[27] is begun by suddenly shifting the $28 \mathrm{MHz}$ phase by 180 degrees for a small fraction of a synchrotron period, and then shifting the phase back for about 3/8 of a synchrotron period, to get an "upright" beam ellipse with the correct aspect ratio. Then the $28 \mathrm{MHz}$ system is snapped off at the same time that the $197 \mathrm{MHz}$ system is snapped on.

Rebucketing is done for two reasons. First, the high frequency system applies a much larger voltage gradient $d V / d s$, so that the bunches can be made much shorter than $\beta^{*}$ (avoiding the hourglass[28] effect), with a reasonably large longitudinal bunch area (to contain longitudinal emittance growth due to IBS). Second, the $197 \mathrm{MHz}$ cavities can not handle the relatively large frequency swing that is necessary to accelerate gold ions from $\gamma \approx 11$ to $\gamma \approx 108$.

If the $28 \mathrm{MHz}$ rf is adiabatically turned off at storage energy, and then the $197 \mathrm{MHz}$ system is adiabatically turned on, then 2520 evenly populated bunches are created. Some of these bunches would have to be removed in order to create a beam abort gap, but this should not be too difficult.

In order to store between 360 and 2520 ion bunches, an alternative to "direct" and "adiabatic" rebucketing must be found. One way is to modify the existing $197 \mathrm{MHz}$ cavities to accept a frequency swing large enough to accelerate gold ions. The feasibility of such a modification has yet to be established, but it would enable the storage of an (essentially) arbitrary number of ion bunches, between 360 and 2520 .

Another way is to develop an rf system at an intermediate frequency. The possibility of developing a special superconducting rf system at a frequency of either $84 \mathrm{MHz}(h=3 \times 360=1080)$ or $113 \mathrm{MHz}$ $(h=4 \times 360=1440)$ has recently re-entered discussion, as a way of combating IBS effects which are particularly bad in longitudinal phase space [29].

\section{A.2 Kickers}

RHIC is currently filled one bunch at a time, from a small number of bunches (for example scenario "B") which have been accelerated in the AGS. The injection kicker therefore has a relatively short rise time, flat top, and fall time, of approximately $90 \mathrm{~ns}, 45 \mathrm{~ns}$, and $90 \mathrm{~ns}$, respectively. 
Large numbers of bunches would naturally be injected into RHIC in "boxcar" fashion - many bunches transferred with the correct spacing at one time. Ideally it would be possible to inject a "bunch train" as long as the AGS circumference into RHIC in one turn of the AGS. New RHIC injection kicker and AGS extraction kicker systems would have to sustain an injection pulse with a long smooth flat top. This would require that the AGS be capable of preparing a bunch train with the appropriate parameters.

So long as the rise and fall times of the injection kicker system are much smaller than the flat top time, their values are not crucial, since the presence of a few "holes" in the bunch structure is inevitable. Some of these holes are necessary and desirable. For example, judiciously placed holes help combat the electron cloud effect (see below) by allowing the clouds to clear. An abort gap approximately $1 \mu$ s long continues to be necessary to accommodate the rise time of the abort kicker. It is possible (although perhaps not likely) that the current abort kicker system will be adequate for a much larger number of bunches.

\section{A.3 Long range beam-beam interactions}

In the current IR optics the beams begin to be magnetically separated in the DX magnet at only 9.80 meters from the IP, before entry into the first quadrupole. They enter separate beam pipes at a crotch 15.70 meters from the IP. This is illustrated in Fig. 5.

Such early separation has the distinct advantage of immunizing RHIC against parasitic long range beam-beam interactions. For example, it has already been recognized that with proton-proton collisions, a crossing angle is only required when 180 or more bunches are stored. A crossing angle may not be required for gold-gold collisions even with 360 bunches [11, 12]. It is conservative to make the total crossing angle $\alpha=7 \sigma^{*}$ when one is required, in which case the tune shift per parasitic crossing is approximately

$$
\left|\Delta Q_{L R}\right|=\frac{2}{7^{2}} \xi \approx .04 \xi
$$

It has been shown that such crossing angles are not difficult with typical RHIC emittances and apertures [12].

Under these conditions it is permissible to have as many as 20 or 30 parasitic collisions per interaction region, since the long range interactions mainly generate tune shifts, not tune spreads, and barely drive nonlinear resonances. Parasitic collisions are spaced by about $0.76 \mathrm{~m}$ with $M=2520$ bunches, leading to about 25 parasitic collisions per IR if the beams begin to be magnetically separated $\pm 10 \mathrm{~m}$ from the IP.

Although long range beam-beam interactions are an important concern, they do not rule out scenarios with even 2520 bunches.

\section{A.4 Cryogenic beam pipe and BPM signal cable heating}

The image current of the beam which flows in the vacuum chamber walls causes resistive heating. This is not a concern in the sections of beam pipe at room temperature, but has the potential to be a serious problem when the heat is deposited at cryogenic temperatures. A maximum average cryogenic heat load of about 0.5 to 1.0 Watt per meter can be tolerated during continuous running in order to stay within the capacity of the RHIC cryogenic refrigerator.

A careful analysis of the issue of "vacuum pipe heating in RHIC" lead to the engineering decision to use stainless steel beam pipes without a copper coating [13]. Fig. 6 shows the results of extending that analysis to the parameter range being considered for RHIC upgrades. The linear power load depends strongly on the RMS Gaussian bunch length, and on the number of bunches. 


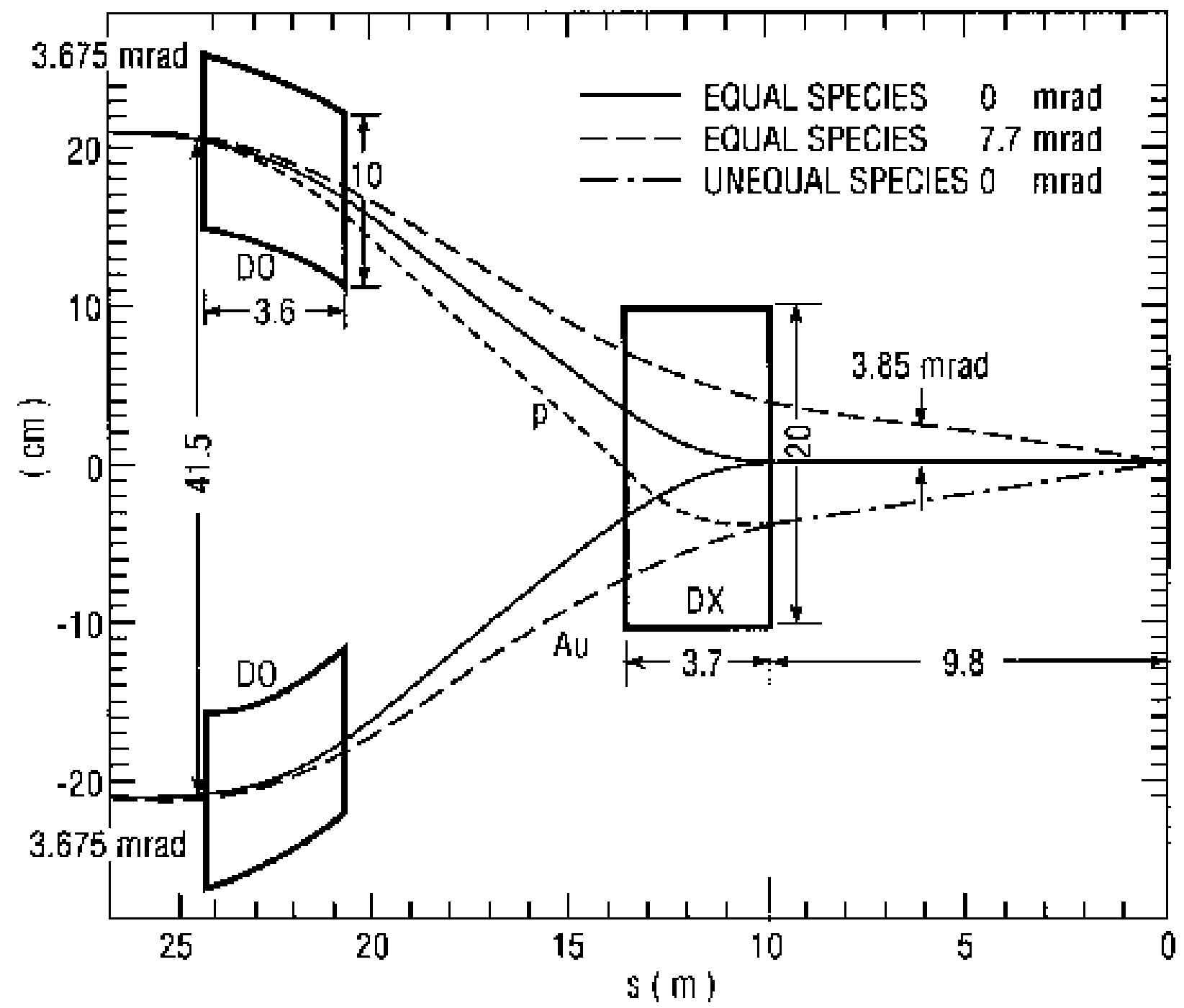

Figure 5: Current layout of the RHIC interaction region, showing a common crossing angle of $3.85 \mathrm{mrad}$ during proton-gold collisions. 

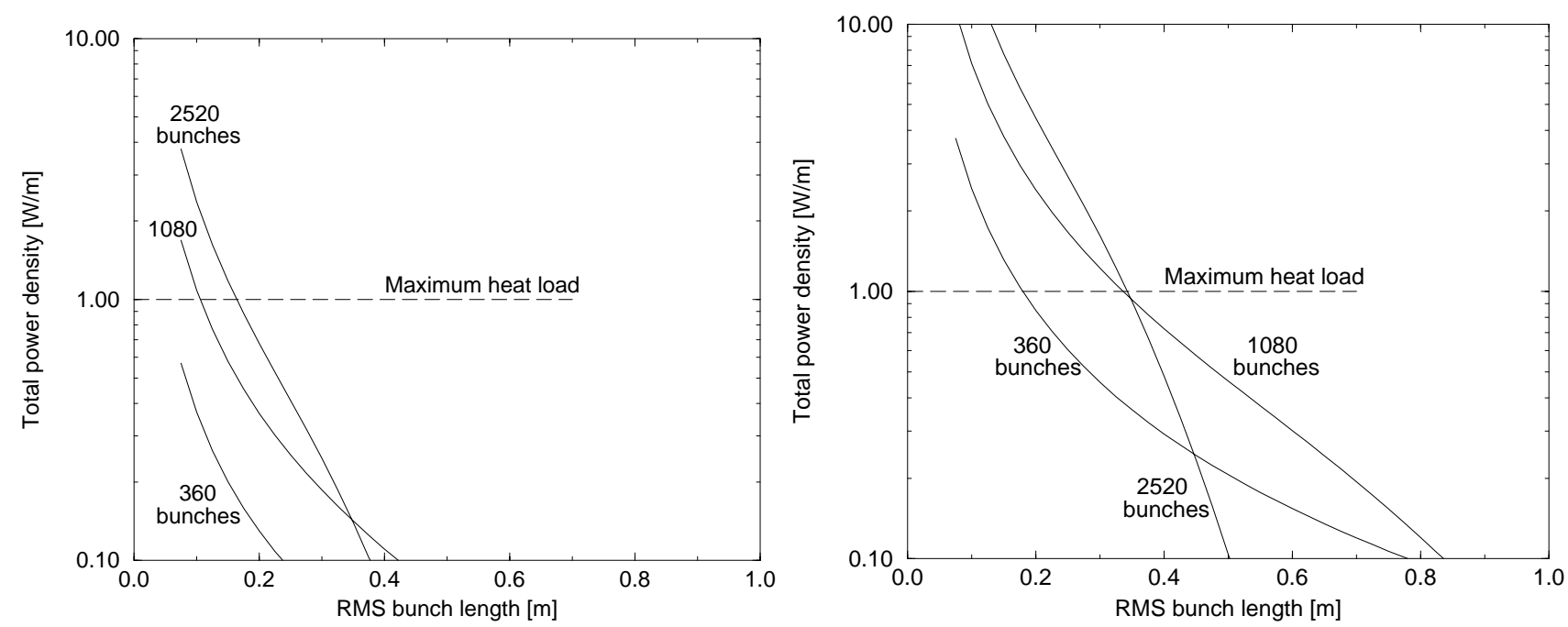

Figure 6: Linear power load deposited at cryogenic temperatures in the stainless steel vacuum chamber, due to beam image currents, with $1 \times 10^{9}$ (on left) and $2.5 \times 10^{9}$ (on right) gold ions per bunch.

The calculation used to generate the data in Fig. 6 naively assumes that all ion bunches have exactly the same charge, and that they are spread uniformly around the circumference (without an abort gap). In this case the power spectrum is a series of narrow lines uniformly spaced by $M f_{\text {rev }}$, under a Gaussian envelope which is the Fourier transform of the bunch shape. The total linear power load is just a sum over all these spectral lines, convoluted with the vacuum chamber resistance at those frequencies - a resistance which is dominated by skin depth effects. As the number of bunches increases, the spacing between spectral lines increases like $M$, but the power in each harmonic increases like $M^{2}$. Thus when the bunches are longitudinally spaced by very many bunch lengths - for example, when $M=360$ - the linear power load is just proportional to $M$, as is intuitively expected.

Fig. 6 shows that this scaling breaks down when there are 2520 bunches in an ion ring, and the bunch spacing is only $1.52 \mathrm{~m}$, except for very short bunch lengths less than, say, $0.25 \mathrm{~m}$. The suppression of the linear power load which is implied for longer bunch lengths is weakened in more realistic situations - for example, when an abort gap is present and when the bunch populations are not all equal. Nonetheless, it is possible to store as many as 2520 bunches in the ion rings without violating the maximum heat load limit, and without losing much luminosity to the hourglass effect[28].

There is also the possibility that the Beam Position Monitor signal cables may suffer unacceptably large heat loads, due to resistive heating by the signal current, when the number of bunches becomes large and the bunches are too short [14]. This problem appears to be less serious than beam pipe heating. It is possible to replace all the signal cables with an upgraded design which is more resilient. In an extreme case the inner diameter of the stainless steel outer conductor of the coaxial line would be copper plated, and the dielectric would be made from silicon dioxide.

\section{A.5 Electron cloud effect}

The electron cloud effect also threatens to overcome the maximum permissible cryogenic heat load in RHIC [15]. In this process akin to multipactor, electrons which are produced by ionization of the residual gas when one bunch passes are attracted and accelerated by the electrical field of the next passing bunch. 
These accelerated electrons eventually hit the vacuum chamber wall and cause the emission of secondary electrons. This process can lead to the runaway accumulation of electrons in the beam pipe, driving a large cryogenic heat load, if the bunches are spaced too closely together.

The effect has been much studied for the LHC, where the nominal bunch spacing is $25 \mathrm{~ns}$ and there are nominally about $10^{11}$ protons per bunch $[16,17,18]$. The recent consensus at CERN appears to be that the electron cloud effect in the LHC is not so dangerous as previously feared [19].

Unfortunately there is a paucity of hard experimental data from existing cryogenic accelerators with closely spaced bunches, although the normal conducting SPS is beginning to generate interesting data with LHC bunch loading parameters [19]. It is noteworthy that in fixed target mode the Tevatron routinely operates with 1008 bunches of approximately $2 \times 10^{10}$ protons, spaced by about $18.9 \mathrm{~ns}(53 \mathrm{MHz})$, without undue cryogenic difficulty. There are 3 missing bunches at 12 locations in the Tevatron bunch train, and an abort gap of about $1 \mu \mathrm{s}$. Such gaps act to clear the electron clouds. The effect with heavy ions may differ from protons, since the cross section for production of the initial electrons has a strong $Z$ depencence.

With $M=360$ the bunches are spaced by $35.6 \mathrm{ns,}$, and with $M=2520$ by $5.1 \mathrm{~ns}$. It is possible that the electron cloud effect will place a hard limit on the number of ion bunches that can be stored in RHIC. This problem needs more investigation, especially in making careful measurements on RHIC, in other cryogenic storage rings - HERA and the Tevatron - and in the SPS.

\section{A.6 Abort dump}

The nominal RHIC beam dump is an internal target, located between the triplet quadrupoles and Q4 on either side of IP10 [8]. As it stands, the dump has a safety factor of about 3 or 4 beyond the "nominal worst case" scenario, in which the carbon-carbon core of the dump is thermally shocked by 60 gold bunches of $10^{9}$ ions, and an emittance of $15 \pi \mu \mathrm{m}$. Clearly, the beam dump needs a radical upgrade if the total current is to be much beyond the "RHIC II" scenario. This should be possible with the addition of more kickers, an extraction septum and a conversion to an external dump system. In principle any amount of stored energy can be aborted, if an elaborate enough external dump is constructed; although, the penalties for misfires may be greatly increased.

Even with an external dump, the downstream Q4 remains vulnerable to quenching through accidental spray during an abort [9]. One relatively simple solution for Q4 would be to reconstruct it as a custom magnet with a beam pipe liner [10].

Additional shielding will be necessary around RHIC, and other more formal requirements will need to be met, in order to raise the Operational Safety Limits and to permit much more intense stored beams.

\section{A.7 Instabilities}

For the higher currents, bunch numbers and beam-beam parameters of the scenarios "A", "B", and "C", we could expect to have problems. Clearly these scenarios will require more study of the impedance and collective instability issues. 


\section{Appendix References}

[1] G. R. Neil et al., Phys. Rev. Lett. 84, 662 (2000).

[2] Phillip J. Bryant and Kjell Johnsen, The Principles of Circular Accelerators and Storage Rings, Cambridge Univ. Press (1993).

[3] Steven D. Holmes, “Tevatron Performance Goals for the Coming Decade", Proc. of the Part. Acc. Conf. 1999, p. 43, New York City (1999).

[4] V. Shiltsev et al., "Compensation of Beam-Beam Effects in the Tevatron Collider with Electron Cooling”, Proc. of the Part. Acc. Conf. 1999, p. 3728, New York City (1999).

[5] E. Keil, R. Talman, Scaling of Luminosity Data between $e^{+} e^{-}$Storage Rings, Part. Acc., 14109 (1983).

[6] S. Peggs, publication under preparation, September 2000.

[7] RHIC Design Manual, http://www.agsrhichome.bnl.gov/NT-share/rhicdm, BNL, 1993.

[8] A.J. Stevens, "Conceptual design of the RHIC dump core”, AD/RHIC/RD-94, BNL, 1995.

[9] A.J. Stevens, "Energy deposition downstream of the internal dump", AD/RHIC/RD-97, BNL, 1995.

[10] A.J. Stevens, private communication, February 2000.

[11] S. Peggs, "Parasitic beam-beam collisions and crossing angles in RHIC", RHIC/AP/66, BNL, 1995.

[12] S. Peggs, "Beam-beam collisions and crossing angles in RHIC" Proc. LHC Beam-Beam Workshop, CERN, April 1999; and RHIC/AP/169, BNL, 1999.

[13] A.G. Ruggiero, S. Peggs, "Vacuum Pipe Heating in RHIC”, RHIC/AP/46, BNL, 1994.

[14] P. Cameron, M. Morvillo, "Thermal Behavior of RHIC BPM Cryogenic Signal Cables” RHIC/AP/68, BNL, 1996.

[15] K.A. Drees, “Beam induced electron clouds at RHIC” RHIC/AP/150, BNL, 1998.

[16] F. Zimmerman, "A simulation study of electron-cloud instability and beam-induced multipacting in the LHC”, LHC Project Report 95, CERN, 1997.

[17] O. Gröbner, "Beam-Induced Electron Cloud in the LHC and Possible Remedies", PAC97, p. 359, Vancouver (1997).

[18] O. Brüning, "Simulations for the beam-induced electron cloud in the LHC liner", LHC Project Report 102, CERN (1997).

[19] L. Evans, "LHC electron cloud key parameters", presentation to the LHC Machine Advisory Committee, unpublished, CERN (1999). 
[20] M. Blaskiewicz et al., "Collective Instabilities in RHIC”, RHIC/AP/36 (1994).

[21] G. Budker, Sov. J. Atomic Energy 22, 346 (1967).

[22] V. V. Parkhomchuk et al., A design study for electron cooling in RHIC is presently underway and a document is being prepared.

[23] Jie Wei, "Evolution of Hadron Beams under Intrabeam Scattering”, Proc. of the 1993 Part. Acc. Conf., 3651 (1993).

[24] M. Bell and J. S. Bell, Part. Acc. 12, 49 (1982).

[25] A. Wolf et. al., NIM A441, 183 (2000).

[26] A. J. Baltz, M. J. Rhoades-Brown, and J. Weneser, Phys. Rev. E 54, 4233 (1996).

[27] “The Conceptual Design of the RHIC RF System”, May 1993.

[28] W. W. MacKay, "Luminosity as Calculated from Machine Parameters", http://www.rhichome.bnl.gov/RHIC/luminosity/lumrep.ps (1999).

[29] M. Brennan, private communication, February 2000. 